\title{
An Intrinsically Digital Amplification Scheme for Hearing Aids
}

\author{
Peter J. Blamey \\ Dynamic Hearing Pty Ltd, 2 Chapel Street, Richmond 3121, Victoria, Australia \\ Email: pblamey@dynamichearing.com.au \\ David S. Macfarlane \\ Dynamic Hearing Pty Ltd, 2 Chapel Street, Richmond 3121, Victoria, Australia \\ Brenton R. Steele \\ Dynamic Hearing Pty Ltd, 2 Chapel Street, Richmond 3121, Victoria, Australia \\ Email:bsteele@dynamichearing.com.au
}

Received 30 April 2004; Revised 23 November 2004

\begin{abstract}
Results for linear and wide-dynamic range compression were compared with a new 64-channel digital amplification strategy in three separate studies. The new strategy addresses the requirements of the hearing aid user with efficient computations on an openplatform digital signal processor (DSP). The new amplification strategy is not modeled on prior analog strategies like compression and linear amplification, but uses statistical analysis of the signal to optimize the output dynamic range in each frequency band independently. Using the open-platform DSP processor also provided the opportunity for blind trial comparisons of the different processing schemes in BTE and ITE devices of a high commercial standard. The speech perception scores and questionnaire results show that it is possible to provide improved audibility for sound in many narrow frequency bands while simultaneously improving comfort, speech intelligibility in noise, and sound quality.
\end{abstract}

Keywords and phrases: amplification, compression, hearing instrument, ADRO.

\section{BACKGROUND}

Although digital hearing aids have been commercially available since 1995 they have not entirely met the high expectations of most hearing aid users. One of the reasons for this is that analog amplification schemes had already reached a high level of sophistication before digital technology was introduced [1]. Most of the advances in digital signal processing have been in the peripheral features of the hearing aid such as multiple programs for different environments, directional microphones, feedback cancellation or suppression, and noise reduction. The basic linear and nonlinear amplification schemes that have been implemented digitally have mostly been based on prior analog technology. Digital technology brought greater flexibility and accuracy to hearing aids, but the first new amplification scheme is the ADRO (adaptive dynamic range optimization) processing strategy.

This is an open access article distributed under the Creative Commons Attribution License, which permits unrestricted use, distribution, and reproduction in any medium, provided the original work is properly cited.

\section{HEARING AID REQUIREMENTS FROM THE USER'S PERSPECTIVE}

The ADRO amplification scheme described below was derived directly from the fundamental requirements for a hearing aid, and was not derived from prior analog amplification systems. These requirements are well described in the Marke Trak surveys of Kochkin [2] and in classic hearing aid texts such as Skinner [3], Valente [4], and Dillon [5]. Kochkin's survey in 2002 revealed that the five most prevalent requirements were as listed below:

(i) $95 \%$ of hearing aid users seek improved listening to speech in noise;

(ii) $88 \%$ of hearing aid users seek better sound quality;

(iii) $85 \%$ of people want their hearing aids to whistle less;

(iv) $83 \%$ of people feel they need to hear more soft sounds;

(v) $81 \%$ of hearing aid users want increased comfort for loud sounds.

Although it features as the fourth most prevalent requirement on the list, the need to amplify soft sounds is clearly 
the most fundamental requirement for a hearing aid that is designed to compensate for hearing loss. The other requirements on the list are not solely direct consequences of hearing loss, but are at least partially the result of the action of the amplification scheme itself. For example, a multichannel compression hearing aid may reduce positive signal-to-noise ratios in noisy conditions by amplifying the (softer) noise more than the (louder) speech within each channel and reducing the peak-to-trough ratios across frequencies.

\section{COMPROMISES IN CONVENTIONAL HEARING AID PROCESSING}

It is interesting to examine why such a fundamental requirement as providing sufficient amplification for soft sounds to be audible is still reported as a problem for users of modern hearing aids. The explanation rests with the phenomenon of recruitment, where the listener's usable dynamic range of hearing is reduced by the dysfunctional auditory system. Recruitment was first reported by Steinberg and Gardner [6] in their psychoacoustic loudness studies on listeners with impaired hearing. A linear amplification circuit with enough gain to make soft sounds audible will often make loud sounds uncomfortable. Steinberg and Gardner [6] suggested using compression as a solution to this potential problem, although it was many years before this suggestion was implemented in a commercial hearing aid. Subsequent research by Fowler [7] resulted in a formal definition of the term recruitment. Recent research by Buus and Florentine [8] suggests that loudness growth is normal for listeners with impaired hearing, even though dynamic range is smaller than that for listeners with normal hearing. In order to compensate for this reduced dynamic range, compression hearing aids apply less gain to loud sounds than to soft sounds, thus reducing the output levels of loud sounds and making them more comfortable. The input/output function is usually predicted from the audiometric thresholds of the listener using one of the fitting schemes that have been developed over recent years $[9,10]$.

It is also interesting to examine the interaction between compression, as a solution for hearing loss, and the other needs of hearing aid users, as identified by Kochkin [2]. Hearing aid users seek improved sound quality. Fast acting compression with high compression ratios can introduce distortion into the output signals, which reduces sound quality. This compounds the problem for the listener, since perceptual sound quality is also reduced by the effect of the hearing loss, which usually includes both an attenuation and a distortion component [11].

The first requirement on Kochkin's list [2], the need to hear better in background noise, is often also a problem for people with normal hearing, but it is made worse by a loss of frequency selectivity in the damaged auditory system [12]. However, the use of compression amplification can also make speech perception in noise more difficult. This occurs because compression can reduce positive signal-to-noise ratios by amplifying the softer noise more than the louder speech, and by reducing the spectral peak-to-trough ratio if a large number of channels are used.
In summary, the use of compression involves a number of compromises: between the audibility of soft sounds and the comfort of loud sounds; between the amount of compression and the sound quality at the output of the hearing aid; and between the amount of compression and the intelligibility of speech in noise. As a result of these trade-offs, compression schemes vary widely from one hearing aid manufacturer to another in terms of the number of frequency channels, the amount of gain applied to soft sounds, the compression threshold and compression ratio, and the time constants with which the compression is applied. These trade-offs are also a subject of much debate in the literature [13].

Many of the advanced features of digital signal processing hearing aids are designed to compensate for the disadvantages of compression. For example, multiple programs can allow the user to choose different parameters for different situations, thus avoiding some of the most adverse compromises. Noise reduction can help to reduce the discomfort of loud noisy sounds without reducing the levels of speech signals. Directional microphones can improve signal-to-noise ratios at the input to the hearing aid, reducing the need for very high compression ratios, and also compensating for the reduction of the signal-to-noise ratio by the compression amplification scheme.

\section{A DIGITAL AMPLIFICATION SCHEME THAT AVOIDS COMPROMISE}

The availability of powerful, low current drain, and miniature digital signal processors (DSP) now makes it possible to address the needs of hearing aid users with completely novel solutions. The ADRO amplification scheme has been developed to address the basic requirements of the hearing aid user directly, using techniques that were not available before the advent of suitable DSP platforms. ADRO processing provides improved audibility for soft sounds and improved comfort for loud sounds in comparison with compression schemes, and does so without degrading sound quality or reducing intelligibility of speech in noise. Indeed, in controlled clinical trials, ADRO processing showed improved sound quality and improved speech intelligibility in noise for listeners, relative to both linear and compression hearing aids. The digital signal processing techniques that comprise the ADRO approach are described below, and a summary of the experimental results obtained in clinical trials is presented.

Like compression, the ADRO processing arose from psychoacoustic studies on loudness $[6,14]$. These psychoacoustic studies [14] investigated the loudness of acoustic and electric signals for listeners who used a hearing aid in one ear and a cochlear implant in the other. A fundamental requirement for these listeners is that a similar range of sounds should be comfortable and audible in both ears so that both ears can contribute information to the overall perception of the sound. Unlike compression, the ADRO amplification scheme does not attempt to compensate for abnormal loudness growth caused by hearing loss. Instead, it places the most informative part of the signal at each frequency into the most 


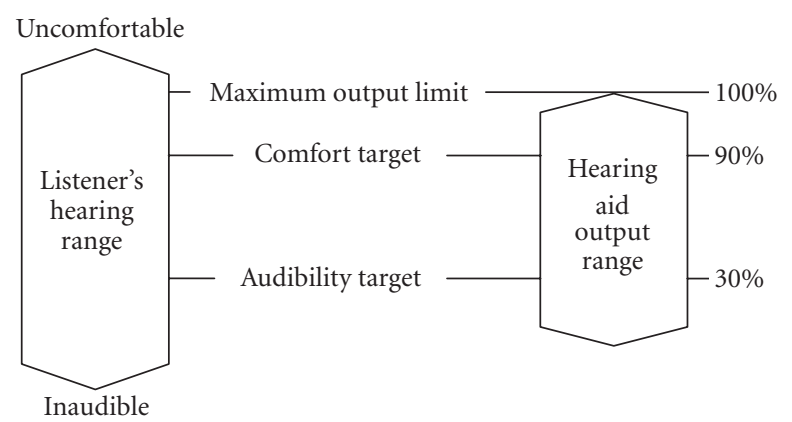

FIgURE 1: The ADRO rules use the maximum output limit, the comfort target, and the audibility target to position the hearing aid output in the optimum part of the listener's hearing range at each frequency.

advantageous part of the listener's hearing range, performing an adaptive dynamic range optimization. The most advantageous part of the hearing range lies within the audible and comfortable region, and it is for this reason that ADRO maximizes intelligibility without compromising comfort or audibility. Because ADRO processing does not just compensate for hearing loss, it has potential applications for normally hearing listeners as well as for listeners with impaired hearing wearing hearing aids, cochlear implants, or both.

\section{THE ADRO RULES}

ADRO uses a set of rules that are applied independently in each frequency channel to keep the output signal in the optimum part of the listener's dynamic range as shown in Figure 1. For a known input signal, such as speech at a given level, a compression scheme could be designed with a frequency-dependent input-output function to place the output signal within the limits shown in Figure 1. Unlike compression, there is no fixed input/output function for the ADRO processing which makes no use of input levels, and therefore ADRO can potentially operate over a wider range of input levels than a compression scheme. The ADRO rules use output targets that are directly controlled by the audiologist during the fitting process without the need to consider complex processing parameters such as compression ratios, knee points, filter shapes, or cross-over frequencies. Unless the ADRO rules are violated, the hearing aid gain will not change in response to a change in input level.

The comfort rule requires the sound to be below the comfort target for $90 \%$ of the time. If the sound is too loud, the gain at that frequency is reduced slowly. The audibility rule requires the sound level to be above the audibility target for $70 \%$ of the time. The audibility rule is checked only if the comfort rule is satisfied. If the sound is too soft, the gain at that frequency is increased slowly. The sizes of the increments and decrements of gain are chosen so that the maximum rate of change is $3 \mathrm{~dB}$ per second. These parameters are approximately equivalent to attack and release times of five seconds or more. The gain in each band is restricted to avoid overamplification of soft background noise and internal noise in the hearing aid. This maximum gain rule also avoids feedback oscillation in quiet situations where the gain might otherwise become very high. Finally, the maximum output rule limits the magnitude of the output level in each frequency band. The maximum output rule operates instantaneously to protect the listener from sudden sounds that would otherwise be too loud. The narrowband maximum output limit reduces the amplitude, leaving the phase unchanged, without introducing spectral distortion into the output signal.

\section{IMPLEMENTATION OF AN ADRO HEARING AID}

The ADRO processing has been implemented on a variety of platforms including the Motorola 56xxx series [15], the Cochlear Limited SPRINT speech processor for cochlear implants [16], the Dspfactory Toccata, and Toccata Plus processors [17]. The ADRO system described below was implemented on the ultra-low-power Toccata Plus hybrid designed by Dspfactory [18]. ADRO uses statistical rules within independent narrow frequency bands and takes the advantage of the discrete Fourier transform (DFT) function of the Toccata Plus weighted overlap add (WOLA) coprocessor. A block diagram of the ADRO processing architecture is shown in Figure 2. At the input to the processing, the samples are buffered into frames (with $50 \%$ overlap), before the WOLA applies a sine (root Hanning) window and performs a discrete Fourier transform. Typically, 64 or 32 frequency bands are used for the ADRO processing in hearing aids. The WOLA then performs the multiplication of the input Fourier components by the gain in each frequency band. The ADRO processing rules are applied independently to each frequency band in the Rcore DSP processor. Finally, the WOLA performs the inverse DFT, windowing, and overlap add functions prior to digital-to-analog conversion and output of the amplified signal to the listener.

With the architecture shown in Figure 2, the implementation of the 64-channel ADRO scheme on Toccata Plus required a power consumption of about $0.8 \mathrm{~mW}$, and fitted comfortably within the available instruction cycles at the 1.28 MHz clock speed. In later implementations, adaptive directional microphone and adaptive feedback detection and suppression algorithms have been added. 


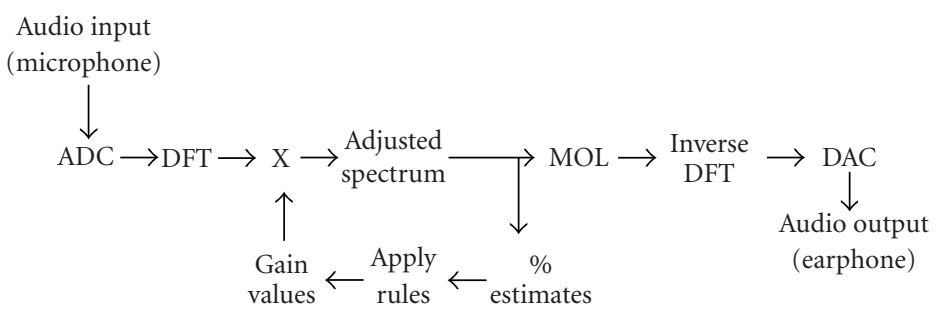

FIgURE 2: Signal processing path for ADRO. Typically, 64 or 32 channels are used in the frequency-domain processing. The abbreviations used are as follows: ADC: analog-to-digital converter; DFT: discrete Fourier transform; X: multiplier; MOL: maximum output limit; DAC: digital-to-analog converter.

\section{STATISTICAL DESCRIPTION OF THE OUTPUT SIGNAL}

The ADRO processing uses estimates of the distribution of output levels in the form of percentiles. For example, the 90th percentile is the level which is exceeded by $10 \%$ of the time, and the 30th percentile is the level that is exceeded by $70 \%$ of the time. These percentiles are estimated by comparing the magnitude of the output amplitude, at each frequency at regular time intervals, with the current value of the percentile estimate. If the magnitude is greater, the estimate is increased by a small amount. If the magnitude is smaller than the estimate, then the estimate is reduced by a small amount. The percentage for each estimator is determined by the ratio of the up and down steps. For example, if the up step is nine times larger than the down step, the estimator will tend to the 90th percentile where the probability of a downward step is nine times greater than the probability of an upward step. The ratio of up and down step sizes is 3:7 for the 30th percentile estimator. The rate at which the percentile estimates change is controlled by the absolute size of the up and down steps and the frequency with which the estimate is updated. Typically, the slew rate for the ADRO percentile estimates is about $20 \mathrm{~dB}$ per second. The comfort rule is implemented by decrementing the gain whenever the 90th percentile rises above the comfort target. The audibility rule is implemented by incrementing the gain whenever the 30th percentile falls below the audibility target. If both rules are satisfied, the gain does not change. The 90th and 30th percentile estimates are calculated independently for each of the ADRO channels (DFT frequencies), and the output of each channel is optimized independently of all other channels.

\section{FITTING THE ADRO TARGETS FOR A HEARING AID USER}

To fit an ADRO hearing aid, the clinician and the client need to establish the audible and comfortable listening range across frequencies. This can be done by measurement or by prediction. The fitting data for the subjects in the initial trials have been analyzed to predict the ADRO parameter values from the audiogram. An alternative fitting prescription is under development, based on the well-established desired sensation level prescription (DSL i/o) [9]. All of the fitting methods share the advantage that the functions of the fitting parameters are easily understood and directly related to the experience of the client when using the hearing aid.

In the clinical trials described below, psychoacoustic measures of comfort were made through the hearing aid. Sixth-octave bands of noise were used with a seven-point scale [19] to establish the dynamic range. The seven categories were: very soft, soft, comfortable but slightly soft, comfortable, comfortable but slightly loud, loud but OK, and uncomfortably loud. The "loud but OK" level was used for the maximum output limit, the "comfortable" level was used for the comfort target, and the audibility target was set equal to the comfort target minus $20 \mathrm{~dB}$ or to threshold, whichever was greater. Finally, the parameters were finetuned for speech and other signals.

The method currently recommended by the authors is a combination of predictive and pragmatic fitting procedures, which is quick to perform and provides an individually tailored fitting. The audiogram is used to predict comfortable levels at seven frequencies spaced at half-octave intervals from $500 \mathrm{~Hz}$ to $4 \mathrm{kHz}$. Noise with 1/6-octave bandwidth is used to balance the loudness across frequency and to check that the overall loudness is towards the upper end of the comfortable range. The comfort targets and other parameters are all predicted from the checked comfortable level measurements and audiometric thresholds. Finally, the overall volume is adjusted for speech signals and the ADRO parameters are fine-tuned according to the preferences of the individual listener.

\section{ADRO HEARING AID TRIALS}

Three medium-scale clinical trials of the 64-channel ADRO strategy have been carried out to provide objective comparisons with linear amplification, three-channel wide dynamic range compression, and a commercial nine-channel wide dynamic range compression scheme. The main features of the studies are listed in Table 1. Complete details may be found in other publications $[15,17]$.

In each of the three studies, the ADRO processing and the comparison amplification scheme were run on identical hardware. Study 1 was conducted with a prototype benchtop 
TABLE 1: Summary of one laboratory study and two field trials of the ADRO hearing aid processing.

\begin{tabular}{|c|c|c|c|}
\hline Study & 1 & 2 & 3 \\
\hline Comparison strategy & Linear with $\mathrm{MOL}^{\mathrm{a}}$ & 3-channel WDRC ${ }^{b}$ & 9-channel WDRC ${ }^{b}$ \\
\hline Processor & Motorola 56003 in laboratory device & Toccata in $\mathrm{BTE}^{\mathrm{c}}$ & Toccata in commercial ITE ${ }^{d}$ \\
\hline Number of subjects & 15 & 19 & 22 \\
\hline Hearing range fitted $\left(\mathrm{PTA}^{\mathrm{e}}\right)$ & 44 to $98 \mathrm{~dB} \mathrm{HL}$ & 33 to $95 \mathrm{~dB} H \mathrm{HL}$ & 25 to $58 \mathrm{~dB} \mathrm{HL}$ \\
\hline Comparison fitting rationale & NAL-RPf & NAL-NL1 ${ }^{g}$ & NAL-NL1 ${ }^{g}$ \\
\hline \multirow[t]{3}{*}{ Time constants } & Not applicable & Attack: $10 \mathrm{~ms}$ & Dual time constants \\
\hline & & Release: $80 \mathrm{~ms}$ & Attack: $10,200 \mathrm{~ms}$ \\
\hline & & & Release: $200,800 \mathrm{~ms}$ \\
\hline
\end{tabular}

${ }^{\mathrm{a}} \mathrm{MOL}$ is maximum output limiting; ${ }^{\mathrm{b}} \mathrm{WDRC}$ is wide dynamic range compression; ${ }^{\mathrm{c}} \mathrm{BTE}$ is behind-the-ear hearing aid; ${ }^{\mathrm{d}} \mathrm{ITE}$ is in-the-ear hearing aid; ${ }^{e}$ PTA is pure tone average threshold at 500, 1000, and $2000 \mathrm{~Hz}$; ${ }^{\mathrm{f}} \mathrm{NAL}-\mathrm{RP}$ is the National Acoustics Laboratory revised prescription [20, 21];

gNAL-NL1 is the National Acoustics Laboratory Nonlinear prescription [10].

device and the subjects were given equal practice time with each scheme in the laboratory. Both studies 2 and 3 were done with wearable commercially available hardware. Study 2 is of particular interest in the context of DSPs in hearing aids because a BTE was programmed with both WDRC and ADRO amplification, allowing the researcher to conduct a blind trial in which the listeners could easily switch from one amplification strategy to the other. The subjects wore the hearing aids for a 6-week acclimatization period before evaluation. In study 3 , a balanced reverse block design was used, and the subjects wore the hearing aids for at least 3 weeks acclimatization before evaluation in each phase of the study.

In each study, speech perception in quiet was tested for presentation levels across the range from soft to loud speech. Typically speech levels are described as "casual," "raised," and "loud" for levels of 55, 65, and $75 \mathrm{~dB}$ SPL, respectively [22]. As expected, the lowest scores were obtained for the lowest input levels. Figure 3 summarizes the results for the lowest input levels which are also the most appropriate to assess audibility differences between the hearing aids. Before evaluation, the volume control was set to the subject's preferred listening level for speech at $65 \mathrm{~dB}$ SPL, and kept fixed at that level for the remainder of the evaluation. This models "real life" where listeners set their hearing aid volume controls for comfortable listening under normal conditions. Figure 3 shows scores for City University of New York (CUNY) sentences [23] in quiet at $55 \mathrm{~dB}$ SPL for study 1 , CUNY sentences in quiet at an individually chosen level of 45 to $65 \mathrm{~dB}$ SPL for study 2, and monosyllabic words [24] at an individually chosen level of 50 to $65 \mathrm{~dB}$ SPL for study 3 . The individually chosen presentation levels in studies 2 and 3 were necessary because of the broad ranges of hearing loss, and thus avoided floor effects for some subjects. The same level was used for each amplification scheme. For each study, the group mean score for the ADRO processing was statistically significantly higher than for the comparison scheme $(\mathrm{t}=7.56, \mathrm{df}=13, P<.001$ for study $1 ; \mathrm{t}=5.16, \mathrm{df}=18$, $P<.001$ for study 2 ; and $\mathrm{t}=3.50, \mathrm{df}=21, P<=.002$ for study 3). The mean differences in scores of $36.4 \%, 14.2 \%$, and $7.9 \%$ are all clinically significant, amounting to at least one additional correct word in every 1 or 2 sentences heard.

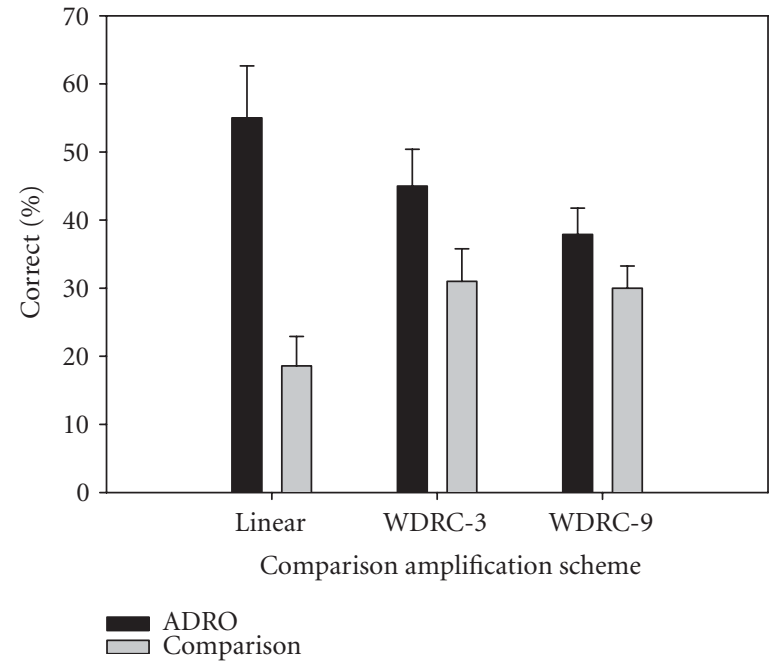

FIGURE 3: Mean scores for perception of soft speech in quiet to assess audibility for the ADRO and comparison amplification schemes in the three studies. Error bars show one standard error of the mean values.

Perception of CUNY sentences in eight-talker babble was also tested in each of the three studies. The presentation level and signal-to-noise ratio was chosen individually for each subject to achieve a score of about $50 \%$ in order to avoid floor and ceiling effects because the groups spanned a wide range of hearing losses. The mean results are shown in Figure 4. For studies 2 and 3, the group mean score was significantly lower for the comparison than for the ADRO processing scheme $(\mathrm{t}$ $=3.80, \mathrm{df}=18, P<=.001$ for study $2 ; \mathrm{t}=2.85, \mathrm{df}=21$, $P<=.01$ for study 3$)$. The mean differences in score were $7 \%, 7.3 \%$, and $7.3 \%$, respectively.

Only study 2 allowed subjects to switch from one amplification scheme to the other quickly while wearing the aids outside the laboratory. This provided an opportunity to do a sensitive preference comparison under realistic conditions. The "hearing aid measure of contrast" (HAMOC) [25] was used. The HAMOC consists of 25 situations in which the listener is asked to choose which of two hearing aids is better, 


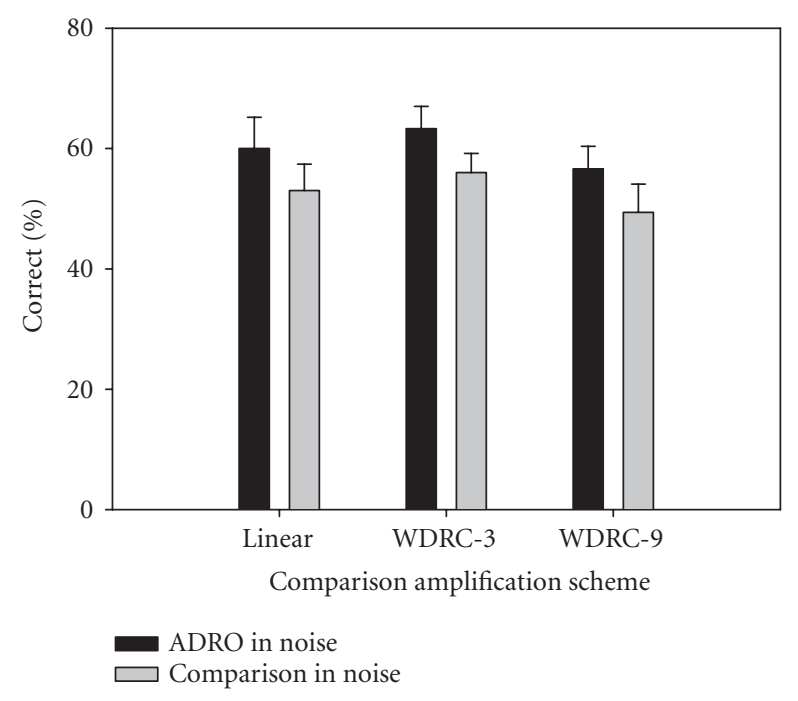

FIGURE 4: Mean scores for speech perception in eight-talker babble to assess intelligibility for the ADRO and comparison amplification schemes in the three studies. Error bars indicate one standard error of the mean values.

and whether it is slightly better, better, or much better than the other. In this blind trial with 19 subjects, ADRO was rated as slightly better in $31 \%$, better in $31 \%$, and much better in $12 \%$ of situations. The total of $74 \%$ preferences compares with $26 \%$ for the three-channel WDRC amplification scheme of which $16 \%$ were slightly better, $10 \%$ were better and $0 \%$ were much better. Chi-squared analysis indicates that the probability of achieving these preference results by chance is less than 0.001 . The pattern of preferences was similar for all situations with most people preferring ADRO in most situations and a few people preferring WDRC in most situations. Overall, three people stated that they preferred WDRC and sixteen preferred ADRO amplification.

\section{DISCUSSION}

The results of the three studies comparing ADRO with linear and WDRC amplification show that clinically and statistically significant improvements to intelligibility of speech at moderately soft levels are possible without compromising comfort, sound quality, or intelligibility in noise. In fact, the ADRO processing was able to produce improvements in all four of these requirements simultaneously. The major factor affecting speech intelligibility at presentation levels of $55 \mathrm{~dB}$ SPL and below is audibility [26]. The data for soft speech in Figure 3 clearly show an intelligibility/audibility difference between ADRO and the comparison amplification scheme, and Figure 4 clearly shows a consistent benefit for speech perception in noise. Studies 2 and 3 were field trials in which the listeners experienced a wide variety of environments and situations over periods of several weeks with each amplification scheme. Their subjective reports and judgments of comfort and sound quality were based on this experience, as sampled by the HAMOC and overall preference questionnaires.
The comparison amplification schemes used in the three studies differ from ADRO, and from one another in several ways: the number of channels used, the time constants (or rate of change of gain), the fitting prescription, and the software implementation on the open-platform DSP processor. Consideration of these differences leads to questions about whether the linear and compression schemes might have performed better if different parameters or fitting prescriptions were used. Although this is possible, it seems unlikely, given that the WDRC and linear amplification schemes and prescriptions are based on years of experience and data from thousands of listeners [10, 20,21]. By contrast, there has been little opportunity for fine-tuning the parameters of ADRO, and there were no fitting data prior to the studies that are described above. The consistent results from three studies with different comparison schemes and spanning a wide range of hearing loss suggest that the ADRO advantages will be robust.

It is also possible that the performance of the ADRO processing could be improved by a different choice of parameters. One example of interest is the use of 32 channels instead of 64. The group delay for ADRO is dependent mainly on the duration and overlap of the windows in the WOLA implementation. For 64 channels at $16 \mathrm{kHz}$ sampling rate and $50 \%$ window overlap, the measured group delay is 12 to $13 \mathrm{~ms}$. For 32 channels, the corresponding delay is $6 \mathrm{~ms}$. Group delay is thought to affect perceived sound quality [27], although there was no indication from the three evaluations that the $13 \mathrm{~ms}$ group delay of the 64-channel system was a problem. The ear molds used in the studies had vents of the minimum size recommended by Dillon [5, pages 144-145] to avoid feedback oscillation and occlusion effects. The reduction from 64 to 32 channels may have other advantages and disadvantages which are unknown at the present time, but informal trials have been encouraging.

Blind trials and comparisons of hearing aid processing schemes in the same hardware are unusual in the literature. One reason for this is the specialized analog and digital ASIC circuits that have been used in the past. The open-platform hearing aid with flexible processing architecture and embedded software provides a new opportunity for studies of this nature. It is likely that ADRO and compression schemes will both improve in the future as controlled studies are carried out to address the questions posed in this discussion section.

In addition to the clinical benefits shown by the studies above, ADRO has other more technical advantages. It utilizes the power and efficiency of the DFT to advantage rather than requiring less-efficient filtering techniques. ADRO calculations are based on output levels which cover a smaller dynamic range than input levels, and thus require less numerical precision. The ADRO parameters (with the exception of maximum gain) are output levels, and so the input microphone can be changed without having to change the fitting targets. For example, a directional microphone, or telecoil, can be used without changing the fitting. This is particularly useful for adaptive directional microphones which can vary in gain and frequency response dynamically [28]. ADRO will adapt dynamically to keep the output levels optimized while 
the microphone is operating to improve signal-to-noise ratios. In addition, the ADRO scheme has advantages to the audiologist because the fitting parameters and methods are quite transparent and intuitive to use. ADRO's narrow frequency bands with minimum overlap and separate adjustments for loud and soft sounds offer high flexibility, with little interaction between the parameters. By contrast, adjustment of one compression parameter such as a compression ratio, kneepoint, or crossover frequency, often requires changes to other parameters because they interact with one another in complex ways.

\section{CONCLUSIONS}

ADRO processing is the first digital amplification scheme designed specifically for the present generation of DSP hearing aids. It uses very efficient digital signal processing techniques and the 64-channel version was easily within the processing capacity of the only available open-platform DSP chip running at its slowest clock speed of $1.28 \mathrm{MHz}$ with a power consumption of less than $0.8 \mathrm{~mW}$. ADRO is an alternative to compression, involving fewer compromises between audibility, comfort, sound quality, and speech intelligibility. Clinical trials of ADRO and WDRC processing in the same processing hardware showed ADRO to be more effective in maintaining an audible and comfortable output signal, and to provide clinically significant improvements in intelligibility and sound quality.

\section{REFERENCES}

[1] L. D. Braida, N. I. Durlach, R. P. Lippman, B. L. Hicks, W. M. Rabinowitz, and C. M. Reed, "Hearing aids-a review of past research on linear amplification, amplitude compression, and frequency lowering," ASHA Monographs, vol. 19, pp. 1-114, 1979.

[2] S. Kochkin, "MarkeTrak VI: consumers rate improvements sought in hearing instruments," The Hearing Review, vol. 9, no. 11, pp. 18-22, 2002.

[3] M. W. Skinner, Hearing Aid Evaluation, Prentice-Hall, Englewood Cliffs, NJ, USA, 1988.

[4] M. Valente, Ed., Strategies for Selecting and Verifying Hearing aid Fittings, Thieme Medical Publishers, New York, NY, USA, 1994.

[5] H. Dillon, Hearing Aids, Boomerang Press, Turramurra, Australia, 2001.

[6] J. C. Steinberg and M. B. Gardner, "The dependence of hearing impairment on sound intensity," Journal of the Acoustical Society of America, vol. 9, pp. 11-23, 1937.

[7] E. P. Fowler, "Some attributes of "loudness recruitment" and "loudness decruitment"," Transactions / American Otological Society, vol. 53, pp. 78-84, 1965.

[8] S. Buus and M. Florentine, "Growth of loudness in listeners with cochlear hearing losses: recruitment reconsidered," Journal of the Association for Research in Otolaryngology, vol. 3, no. 2, pp. 120-139, 2002.

[9] L. Cornelisse, R. Seewald, and D. Jamieson, "The input/output formula: a theoretical approach to the fitting of personal amplification devices," Journal of the Acoustical Society of America, vol. 97, no. 3, pp. 1854-1864, 1995.

[10] NAL-NL1, NAL non-linear User Manual Version 1.1., National Acoustic Laboratories: Chatswood, NSW, Australia, 1999.
[11] R. Plomp, "Auditory handicap of hearing impairment and the limited benefit of hearing aids," Journal of the Acoustical Society of America, vol. 63, pp. 533-549, 1978.

[12] B. Moore and B. Glasberg, "Comparisons of frequency selectivity in simultaneous and forward masking for subjects with unilateral cochlear impairments," Journal of the Acoustical Society of America, vol. 80, pp. 93-107, 1986.

[13] H. Dillon, "Tutorial: Compression? Yes, but for low or high frequencies, for low or high intensities, and with what response times?" Ear and Hearing, vol. 17, no. 4, pp. 287-307, 1996.

[14] P. J. Blamey, G. J. Dooley, C. J. James, and E. S. Parisi, “Monaural and binaural loudness measures in cochlear implant users with contralateral residual hearing," Ear and Hearing, vol. 21, no. 1, pp. 6-17, 2000.

[15] L. Martin, P. J. Blamey, C. J. James, K. L. Galvin, and D. Macfarlane, "Adaptive dynamic range optimisation for hearing aids," Acoustics Australia, vol. 29, no. 1, pp. 21-24, 2001.

[16] C. J. James, P. J. Blamey, L. Martin, B. Swanson, Y. Just, and D. Macfarlane, "Adaptive dynamic range optimization for cochlear implants: a preliminary study," Ear and Hearing, vol. 23, pp. 49S-58S, 2002.

[17] P. J. Blamey, L. Martin, and H. Fiket, "A digital processing strategy to directly optimize hearing aid outputs," Journal of the American Academy of Audiology, vol. 15, pp. 714-726, 2004.

[18] R. Brennan and T. Schneider, "A flexible filterbank structure for extensive signal manipulations in digital hearing aids," in Proc. IEEE Int. Symp. Circuits and Systems (ISCAS '98), vol. 6, pp. 569-572, Monterey, Calif, USA, 1998.

[19] D. M. Hawkins, B. E. Walden, A. Montgomery, and R. A. Prosek, "Description and validation of an LDL procedure designed to select SSPL90," Ear and Hearing, vol. 8, pp. 162-169, 1987.

[20] D. Byrne and H. Dillon, “The National Acoustics Laboratory (NAL) new procedure for selecting the gain and frequency response of a hearing aid," Ear and Hearing, vol. 7, pp. 257-265, 1986.

[21] D. Byrne, A. Parkinson, and P. Newall, "Hearing aid gain and frequency response requirements for the severely/profoundly hearing impaired," Ear and Hearing, vol. 11, pp. 40-47, 1990.

[22] K. S. Pearsons, R. L. Bennett, and S. Fidell, "Speech levels in various noise environments," EPA report 600/1-77-025, Environmental Protection Agency, Washington, DC, USA, 1977.

[23] A. Boothroyd, L. Hanin, and T. Hnath, "A sentence test of speech perception: reliability, set equivalence, and short term learning," Speech \& Hearing Science Report RC10, The City University New York, New York, NY, USA, 1985.

[24] B. Henry, H. J. McDermott, C. M. McKay, C. J. James, and G. M. Clark, "A frequency importance function for a new monosyllable word test," Australian Journal of Audiology, vol. 20, pp. 79-86, 1998.

[25] H. Dillon, A. James, and J. Ginis, "Client oriented scale of improvement (COSI) and its relationship to several other measures of benefit and satisfaction provided by hearing aids," Journal of the American Academy of Audiology, vol. 8, pp. 2743, 1997.

[26] C. V. Hudgins, J. E. Hawkins, J. E. Karlin, and S. S. Stevens, "The development of recorded auditory tests for measuring hearing loss for speech," The Laryngoscope, vol. 57, pp. 57-89, 1947.

[27] J. Agnew and J. M. Thornton, "Just noticeable and objectionable group delays in digital hearing aids," Journal of the American Academy of Audiology, vol. 11, no. 6, pp. 330-336, 2000. 
[28] T. Fortune, "Adaptive dynamic range optimization with beamforming: An interesting combination," in 49th International Congress of Hearing Aid Acousticians, Frankfurt am Main, Germany, 2004.

Peter J. Blamey graduated from the Australian National University in 1972, where he was awarded the University Medal in theoretical physics. He later gained a Ph.D. degree in physics from Monash University. Dr. Blamey was the Inventor and Leader of the Combionic Aid team that initially developed the ADRO strategy at the Cooperative Research Centre for Cochlear Implant and Hearing Aids. During his career as the Principal Research Fellow within the Department of Otolaryngology at the University of Melbourne, Dr. Blamey has worked on the bionic ear, hearing aids, and other devices for people with impaired hearing. He has published over 130 papers in physics, psychophysics, speech processing, speech perception, and language development in children with impaired hearing. Dr. Blamey is an inventor of 10 international patents or patent applications, including ADRO. He has made a major contribution to improving speech and language skills in deaf children.

David S. Macfarlane graduated from the Electronic Engineering Department of La Trobe University with first-class honours. He was awarded the University Medal from the Friends of the University for achieving the highest results in the Physical Sciences Faculty. Macfarlane also has a Bachelor of Music from Sydney University. Macfarlane has developed electronic hardware and software and provided engineering support for the ADRO processing scheme since 1997.

Brenton R. Steele received first-class honours when he graduated from Monash University with a Bachelor of Electrical and Computer Systems Engineering. During his Ph.D. research he developed a new, efficient digital filter architecture and has published several papers on the topic. Dr. Steele gained his Ph.D. degree in 2003 from RMIT University and has had articles published in eight peer-reviewed journals. His work with

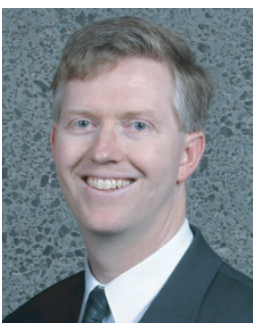
ADRO has involved developing and implementing digital signal processing algorithms on low-power processors.

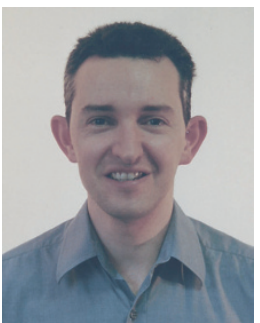

